\title{
Risk Factors for Nosocomial Infection in a Brazilian Neonatal Intensive Care Unit
}

\author{
Ana Carolina Vieira Costa Fernandes Távora ${ }^{1}$, Antonieta B. Castro ${ }^{2}$, Maria Afonsina M. Militão ${ }^{2}$, José Eduilton Girão ${ }^{2}$, Karina de \\ Cássia Braga Ribeiro ${ }^{3}$ and Lara Gurgel Fernandes Távora ${ }^{2}$ \\ ${ }^{1}$ Walter Cantídeo Universitary Hospital; ${ }^{2}$ César Cals Hospital; ${ }^{3}$ ACCamargo Cancer Hospital; São Paulo, SP, Brazil
}

\begin{abstract}
This study was designed to describe the epidemiology and risk factors for nosocomial infection (NI) in a Brazilian neonatal intensive care unit (NICU). This study was a retrospective cohort from January to December, 2003. All neonates admitted to the NICU. Infection surveillance was conducted according to the NNIS, CDC. Chi-square test and logistic regression model were performed for statistical analyses. The study was conducted at a public, tertiary referral NICU of a teaching hospital in the Northeast of Brazil. A total of 948 medical records were reviewed. Overall $\mathrm{NI}$ incidence rate was $34 \%$. The main neonatal NI was bloodstream infection $(68.1 \%)$, with clinical sepsis accounting for $47.2 \%$, and pneumonia was the second most common NI (8.6\%). Multivariate analysis identified seven independent risk factors for NIs: birth weight, exposure to parenteral nutrition, percutaneous catheter, central venous catheter or mechanical ventilation, abruptio placentae and mother's sexually transmitted disease (STD). Neonates from mothers with STD or abruptio placentae, those weighing less than $1,500 \mathrm{~g}$ at birth or those who used invasive devices were at increased risk for acquiring NI.
\end{abstract}

Key-Words: Risk factors, neonatal nosocomial infection, neonatal intensive care unit.

Despite intensive surveillance and prophylactic measures, nosocomial infections (NIs) in neonatal intensive care units (NICUs) remain a major world health problem. NIs still occur at an incidence of around 30\% [1,2] and, in developing countries, are estimated to cause $40 \%$ of all neonatal deaths [3].

At birth, newborns are devoiced of efficient structural barriers, of a protective endogenous microbial flora and of a mature immune system. Additionally, neonates admitted to NICUs, especially if premature or with low birth weight, are under a profound physiologic instability and are frequently exposed to therapeutic interventions associated with infectious complications, such as invasive procedures and broad-spectrum antibiotics [4,5]. Together, these unique aspects render this population exceedingly susceptible to NIs, as well as vulnerable to more severe illness.

The outcome of neonates with NICU NIs is strongly related to their appropriate diagnosis and management. Diagnosing neonatal NI, however, is a challenge, since clinical signs and symptoms are often nonspecific for a particular infection. As a consequence, deciding whether to treat or not, balancing optimal patients care with aspects such as possible adverse events or antibiotic resistance, may be difficult. In line with this idea, the recognition of the epidemiology and risk factors for neonatal NI is extremely relevant in the clinical setting, since it contributes to the diagnostic reasoning and supports clinical decisions. Also, this knowledge offers the target to control strategies that may minimize the morbidity, mortality and, consequently, the high costs associated with hospitalacquired infections.

Received on 22 July 2007; revised 22 January 2008.

Address for correspondence: Dr.Lara Gurgel Fernandes Távora. 482, Professor Dias da Rocha street apt. 34. Phone: 85 3261-4671 / 85 9973-0656 / 85 8886-3412. Zip code 60170-310. Fortaleza, CE, Brazil. E-mail: laratavora@gmail.com.

The Brazilian Journal of Infectious Diseases 2008;12(1):75-79. (C) 2008 by The Brazilian Journal of Infectious Diseases and Contexto Publishing. All rights reserved.
Brazilian data concerning NICU NI are limited and most of the information comes from international experience. The purpose of this study was to describe the epidemiology and risk factors for NI in a Brazilian NICU.

\section{Material and Methods}

The study was conducted at a public, 22-bed NICU of a general teaching hospital and tertiary care referral center in the Northeast of Brazil. The 22 beds are divided in two rooms, one with ten beds and the other with 12 . In each room, there are three hand-washing sinks. The ratio nurse-to-patient per NICU room is 1:5. A protocol based on recent literature and discussed with the hospital infection surveillance group is used for antibiotic prescription. Such group is available for consults whenever necessary.

This retrospective cohort included all the neonates admitted to the NICU from January 1st to December 31st, 2003. Their medical records were reviewed until discharge from the unit or death. For each patient, data on birth weight, adequacy for gestational age [6,7], gender, Apgar score at one and five minutes, neutrophil count at 12 hours of life, medical devices used (central venous catheter, umbilical catheter, percutaneous catheter, total parenteral nutrition, mechanical ventilation), other relevant medical conditions and length of stay were collected. The mother's medical records were also analyzed with respect to maternal age, chronic medical conditions, past pregnancy history, antenatal care, pertinent aspects of the social history, peripartum events of importance and intrapartum assistance.

Maternal age was arranged in quartiles: $<19$ years, 19-23 years, 24-29 years and $>29$ years. A positive history for antenatal care was defined as $\geq 4$ antenatal visits [8]. Gestational age was classified into five distinct groups: $<29$ weeks, 29-33 weeks, 34-36 weeks, 37-42 weeks, $>42$ weeks. Birth weight categories were: $\leq 1,000 \mathrm{~g}, 1,001-1,500 \mathrm{~g}, 1,501$ $2,500 \mathrm{~g},>2,500 \mathrm{~g}$. Newborns were stratified based on three Apgar score groups: 0-3, 4-6, 7-10 [9]. Twelve-hour neutrophil 
counts in the neonate were categorized as low $(<7,800)$, adequate $(7,800-14,400)$ or high $(>14,400)[10]$.

Infection surveillance was consistently conducted according to the National Nosocomial Infection Surveillance System (NNIS/CDC/Atlanta) definitions, which consider all neonatal infections, whether acquired during delivery or during hospitalization, as nosocomial, unless evidence indicates transplacental acquisition [11,12]. Bloodstream infections were considered as clinical sepsis when clinical and laboratory findings of infection were present, without positive cultures, and as confirmed when positive cultures were also present. Catheter related bloodstream infection was defined as the isolation of the same microbe from blood cultures that is shown to be significantly colonizing the catheter of a patient with clinical features of bloodstream infection in the absence of any other local infection caused by the same microbe that could have given rise to bloodstream infection [13]. Nosocomial pneumonia required new sputum production, progressive new infiltrate not present on admission chest radiograph accompanied by respiratory failure and laboratory evidence of infection. Surgical site infections was diagnosed on the basis of one of the following: purulent drainage from incision site or from a drain; positive result from a culture of fluid obtained from a surgical site closed primarily; the surgeon's or attending physician's diagnosis of infection; or the surgical site requires reopening.

\section{Statistical Analysis}

Descriptive statistics was performed for all the studied variables. Some of them were then categorized according to the frequency analysis. Chi-square test was performed for the association between potential risk factors and NIs. The variables with $p<0.20$ in the univariate analyses were included in multivariate logistic regression model in order to identify independent risk factors for NICU NI. The level of statistical significance adopted was $\mathrm{p}<0.05$.

SPSS for Windows 10.0 software (Chicago, IL, USA) was used for all statistical analysis.

\section{Results}

Nine hundred and forty-eight patients were documented during the study period. Table 1 shows the patients' profile.

A total of 244 patients developed 324 NIs (19.4\% of patients had only one infection and $6.3 \%$ of patients had more than one infection). The total NI incidence rate was $34 \%$. Bloodstream infection was the most frequent NI (68.2\%), with clinical sepsis accounting for $47.2 \%$. Pneumonia ranked second (8.6\%), followed by omphalitis (4.3\%), surgical site infection (2.4\%) and impetigo (1.2\%). Fifty patients (15.4\%) had other infections, mainly represented by meningitis and eye, mouth or skin infections, especially mucocutaneous candidiasis (Table 2).

The variables associated with NI according to the univariate analysis were: delivery complications $(\mathrm{p}=0.03)$, mother's sexually transmitted diseases (STD) $(\mathrm{p}=0.04)$, antenatal care $(\mathrm{p}<0.01)$, premature rupture of membranes $(\mathrm{p}=$ $0.01)$, abruptio placentae $(\mathrm{p}<0.01)$, gestational age $(\mathrm{p}<0.01)$, birth weight $(p<0.01)$, first minute Apgar score $(p=0.04)$, number of invasive devices ( $>2$ devices) $(\mathrm{p}<0.01)$, percutaneous catheter $(\mathrm{p}<0.001)$, central venous catheter $(\mathrm{p}<$ $0.001)$, mechanical ventilation $(\mathrm{p}<0.001)$, umbilical catheter $(\mathrm{p}<0.01)$, total parenteral nutrition $(\mathrm{p}<0.01)$ and length of stay $(\mathrm{p}<0.01)$ (Table 1). The following variables, although not statistically significant $(\mathrm{p}<0.05)$, were included in the multivariate analysis $(\mathrm{p}<0.20)$ : maternal hypertension $(\mathrm{p}=0.12)$, premature delivery $(\mathrm{p}=0.10)$, meconial bronchoaspiration $(\mathrm{p}=$ $0.10)$, fifth minute Apgar score ( $\mathrm{p}=0.18)$ and neutrophil count $(\mathrm{p}=0.06)$.

The multivariate analysis identified seven independent risk factors for NIs in the NICU: mother's $\operatorname{STD}(\mathrm{p}=0.02$, OR 4.03 ; $95 \%$ CI 1.23-13.11), abruptio placentae ( $\mathrm{p}<0.01$, OR 2.73 ; 95\% CI 1.38-5.40), birth weight $<1000 \mathrm{~g}$ ( $\mathrm{p}=0.01$; OR $1.99,95 \%$ CI 1.17-3.36), birth weight 1001-1500 g ( $<<0.01$; OR $2.24,95 \%$ CI 1.41-3.54), exposure to parenteral nutrition ( $\mathrm{p}<$ $0.01, \mathrm{OR}=6.35 ; 95 \%$ CI 4.14-9.75), percutaneous catheter $(\mathrm{p}<$ 0.01 , OR 10.88 ; 95\% CI 3.21-36.83), central venous catheter $(\mathrm{p}<0.01$, OR 4.32; 95\% CI 1.95-9.56) and mechanical ventilation $(\mathrm{p}<0.01$, OR 3.42; 95\% CI 2.17-5.41) (Table 3).

\section{Discussion}

Especially in NICUs, NI is recognized as one of the most significant causes of morbidity and mortality among hospitalized newborns [2]. However, the exact impact of this condition is difficult to point out, since there is a wide variation in infection rates reported in the literature, possibly due to differences in surveillance or study methods. In an attempt to overcome this problem, this study adopted NNIS definitions. Around the world, each NICU has unique characteristics that are reflected in the epidemiology of NIs. Obviously then, it is extremely important to control the inherent aspects of each NICU, and to make it available to the local laborious body and the scientific community interested in epidemiological data. Unfortunately, this practice is still not universal, and there are not many published studies that portray the epidemiology and risk factors for NI in Brazilian NICUs.

In this series, the overall NI incidence rate observed (34\%) is approximately the mean of the recently reported Brazilian incidence rates (18.9\% [2], 50.7\% [14] and 22\% [1]).

Bloodstream infection was the most prevalent infection in this survey, with clinical sepsis accounting for the majority of cases, and nosocomial pneumonia was the second most prevalent one. This distribution is similar to that reported by other authors $[1,5,15,16]$, although different from some Brazilian reports $[2,14]$, which describe pneumonia as the most common neonatal NI. The proportion of bloodstream infections in this study $(68.2 \%)$ is definitely worrisome, since neonatal sepsis carries on a particular increased mortality, prolonged length of hospital stay and slower growth among very low birth weight infants and our rates are higher than those usually observed [1,3,5,15]. 
Table 1. Distribution of 948 patients according to clinical and demographic variables and the occurrence of NI, NICU - Hospital Dr. César Cals, January to December, 2003

\begin{tabular}{|c|c|c|c|c|}
\hline \multirow{2}{*}{ Variable } & & \multicolumn{2}{|c|}{ Total* (n) (\%) } & \multirow{2}{*}{$\mathbf{p}$} \\
\hline & & With NI & Without NI & \\
\hline \multirow[t]{2}{*}{ Gender } & Male & $134(26.0)$ & $382(74.0)$ & 0.65 \\
\hline & Female & $107(27.3)$ & $285(72.7)$ & \\
\hline \multirow[t]{4}{*}{ Maternal age (years) } & $<19$ & $65(26.7)$ & $178(73.3)$ & 0.31 \\
\hline & $19-23$ & $56(28.7)$ & 139 (71.3) & \\
\hline & 24-29 & $65(29.5)$ & $155(70.5)$ & \\
\hline & $>29$ & $47(22.2)$ & $165(77.8)$ & \\
\hline \multirow[t]{2}{*}{ Antenatal care (visits) } & Yes $(\geq 4)$ & $98(22.3)$ & $342(77.7)$ & $<0.01$ \\
\hline & No $(<4)$ & 119 (31.2) & $263(68.8)$ & \\
\hline \multirow[t]{3}{*}{ Rupture of membranes } & No & $156(29.2)$ & $378(70.8)$ & 0.02 \\
\hline & $<24 \mathrm{~h}$ & $33(23.7)$ & $106(76.3)$ & \\
\hline & $\geq 24 \mathrm{~h}$ & $35(19.4)$ & $145(80.6)$ & \\
\hline \multirow[t]{2}{*}{ Delivery } & Cesarean & $131(26.3)$ & $367(73.7)$ & 0.63 \\
\hline & Vaginal & $113(27.7)$ & $295(72.3)$ & \\
\hline \multirow[t]{4}{*}{ Gestational age (weeks) } & $<29$ & $43(47.3)$ & $48(52.7)$ & $<0.01$ \\
\hline & $29-33$ & 137 (33.2) & $276(66.8)$ & \\
\hline & $34-36$ & $35(14.8)$ & $201(85.2)$ & \\
\hline & $37-42$ & $21(14.4)$ & $125(85.6)$ & \\
\hline \multirow[t]{2}{*}{ Maternal disease } & Yes & $177(28.1)$ & $452(71.9)$ & 0.19 \\
\hline & No & $55(23.7)$ & $177(76.3)$ & \\
\hline \multirow[t]{2}{*}{ Maternal hypertension } & Yes & $56(23.2)$ & $185(76.8)$ & 0.12 \\
\hline & No & $176(28.4)$ & 444 (71.6) & \\
\hline \multirow[t]{2}{*}{ Abruptio placentae } & Yes & $27(45.8)$ & $32(54.2)$ & $<0.01$ \\
\hline & No & $204(25.5)$ & $597(74.5)$ & \\
\hline \multirow[t]{2}{*}{ Premature delivery } & Yes & $74(31.0)$ & $165(69.0)$ & 0.10 \\
\hline & No & $158(25.4)$ & 464 (74.6) & \\
\hline \multirow[t]{4}{*}{ Birth weight (g) } & $\leq 1,000$ & $75(49.7)$ & $76(50.3)$ & $<0.01$ \\
\hline & $1,001-1,500$ & $86(37.2)$ & $145(62.8)$ & \\
\hline & $1,501-2,500$ & $60(16.5)$ & $304(83.5)$ & \\
\hline & $>2500$ & $23(13.1)$ & $152(86.9)$ & \\
\hline \multirow[t]{3}{*}{ Adequacy for gestational age } & SGA & $45(28.7)$ & $112(71.3)$ & 0.28 \\
\hline & AGA & $149(26.5)$ & $414(73.5)$ & \\
\hline & LGA & $3(13.0)$ & $20(87.0)$ & \\
\hline \multirow[t]{3}{*}{ First minute Apgar score } & $0-3$ & $57(27.9)$ & $147(72.1)$ & 0.04 \\
\hline & $4-6$ & $84(31.8)$ & $180(68.2)$ & \\
\hline & $7-10$ & $97(23.2)$ & $321(76.8)$ & \\
\hline \multirow[t]{3}{*}{ Fifth minute Apgar score } & $0-3$ & $4(18.2)$ & $18(81.8)$ & 0.18 \\
\hline & $4-6$ & $42(33.1)$ & $85(66.9)$ & \\
\hline & $7-10$ & $192(26.3)$ & $538(73.7)$ & \\
\hline \multirow[t]{2}{*}{ Meconium aspiration } & Yes & $11(18.0)$ & $50(82.0)$ & 0.10 \\
\hline & No & $212(27.6)$ & $555(72.4)$ & \\
\hline \multirow[t]{3}{*}{ Neutrophil count } & $<7,800$ & 131 (30.3) & $301(69.7)$ & 0.06 \\
\hline & $7,800-14,400$ & $51(23.2)$ & $169(76.8)$ & \\
\hline & $>14,400$ & $32(34.8)$ & $60(65.2)$ & \\
\hline \multirow[t]{3}{*}{ Invasive devices (number) } & 0 & $27(6.0)$ & $421(94.0)$ & $<0.01$ \\
\hline & $1-2$ & $82(27.8)$ & $213(72.2)$ & \\
\hline & $>2$ & $134(77.0)$ & $40(23.0)$ & \\
\hline \multirow[t]{3}{*}{ Length of hospitalization (days) } & $\leq 4$ & $19(4.2)$ & $433(95.8)$ & $<0.01$ \\
\hline & $5-11$ & $81(32.0)$ & $172(68.0)$ & \\
\hline & $>11$ & $144(65.8)$ & 75 (34.2) & \\
\hline
\end{tabular}

NI: Nosocomial infection; NICU: neonatal intensive care unit. * Excluded patients (missing values): gender (24 patients); maternal age (78 patients); antenatal care (128 patients); rupture of membranes (95 patients); delivery type (42 patients); gestational age (62 patients); maternal disease (87 patients); maternal hypertension (87 patients); abruptio placentae (88 patients); premature delivery (87 patients); birth weight (27 patients); category (207 patients); APGAR 1' (62 patients); APGAR 5' (69 patients); meconium aspiration (120 patients); neutrophil count (204 patients); invasive devices (31 patients); length of hospitalization (24 patients). 
Table 2. Neonatal Nosocomial Infection Site Distribution Hospital Dr César Cals, January to December, 2003

\begin{tabular}{lcc}
\hline Localization & N & Percent \\
\hline Bloodstream infection & 221 & $68.2 \%$ \\
$\quad$ With positive cultures & 68 & $20.9 \%$ \\
$\quad$ Clinical sepsis & 153 & $47.2 \%$ \\
Pneumonia & 28 & $8.6 \%$ \\
Omphalitis & 14 & $4.3 \%$ \\
Surgical site infection & 8 & $2.4 \%$ \\
Impetigo & 3 & $1.2 \%$ \\
Others & 50 & $15.4 \%$ \\
Total & 324 & $100 \%$ \\
\hline
\end{tabular}

Table 3. Independent risk factors for NICU-acquired infection (logistic regression analysis) - Hospital Dr César Cals, January to December, 2003

\begin{tabular}{lccc}
\hline Variable & $\begin{array}{c}\text { Odds } \\
\text { ratio }\end{array}$ & $\begin{array}{c}\text { 95\% CI } \\
\text { adjusted OR }\end{array}$ & p \\
\hline Mother's STD & 4.03 & $1.23-13.11$ & 0.02 \\
Abruptio placentae & 2.73 & $1.38-5.40$ & $<0.01$ \\
Birth weight $<$ 1000 g & 1.99 & $1.17-3.36$ & 0.01 \\
Birth weight 1001-1500g & 2.24 & $1.41-3.54$ & 0.01 \\
Parenteral nutrition & 6.35 & $4.14-9.75$ & $<0.01$ \\
Percutaneous catheter & 10.88 & $3.21-36.83$ & $<0.01$ \\
Central venous catheter & 4.32 & $1.95-9.56$ & $<0.01$ \\
Mechanical ventilation & 3.42 & $2.17-5.41$ & $<0.01$ \\
\hline
\end{tabular}

Delivery complications, antenatal care, premature rupture of membranes, gestational age, first minute Apgar score, number of invasive devices ( $>2$ devices), umbilical catheter and length of stay were associated with NI in the univariate analysis but were not statistically significant in the logistic regression model.

The multivariate analysis identified three groups of independent risk factors for neonatal NICU-acquired infection: maternal factors (mother's STD and abruptio placentae), intrinsic neonatal risk factors (birth weight < $1500 \mathrm{~g}$ ) and extrinsic neonatal risk factors (exposure to parenteral nutrition, percutaneous catheter, central venous catheter and mechanical ventilation).

Maternal infection has already been described as an independent risk factor for neonatal NI [2]. Abruptio placentae is a serious condition with a high mortality rate; liveborn infants frequently have neonatal complications, including birth asphyxia and the hazards of prematurity, what rises the need for medical intervention. In this study, although the number of antenatal visits was not linked with an increased risk of neonatal infection, the results strengthened requirements for a better-quality antenatal care, since both maternal risk factors could be, at least in part, avoided with this practice.
Birth weight has been consistently considered as a strong and independent predictor of adverse outcomes, including NIs $[1,2,4,14]$. In this study, while the odds ratio of infants whose birth weights were 1001-1500 g was 2.24, which is in accordance with previously published data [4], newborns with a birth weight $<1,000 \mathrm{~g}$ had an odds ratio of 1.99 . Given that these newborns are often more severely ill, the majority of them die before the NI is documented or even before it really happens. This may explain the apparent paradox of the statistical result. It also underlines the limited ability of our NICU in changing the outcome of these extremely low birth weight newborns.

Devices are part of the advances in medical therapy that have resulted in significant improvements in neonatal survival. On the other hand, it is well recognized that these same beneficial tools can also place the newborn at a considerable higher risk of health-care associated infections [1,2,4,14]. In this series, the exposures to parenteral nutrition, percutaneous catheter, central venous catheter or mechanical ventilation independently increased the risk for neonatal NIs.

In conclusion, this study showed that mother's STD, abruptio placentae, birth weight $<1500 \mathrm{~g}$ and exposure to parenteral nutrition, percutaneous catheter, central venous catheter and mechanical ventilation are independent risk factors for NICU- acquired infections. These results raise two important matters: first, the necessity of providing a better antenatal care and avoid the occurrence of complications secondary to mother's factors; second, the implementation of protocols for judicious use of invasive procedures on NICUs. We believe that these actions together will definitely decrease the incidence of neonatal nosocomial infections in our institution. Furthermore, the knowledge of prognostic factors for neonatal NI allows a precise stratification of the population at risk and the implementation of more efficient and tailored therapeutic strategies.

\section{References}

1. Pessoa-Silva C.L., Richtmann R., Calil R., et al. Healthcareassociated infections among neonates in Brazil. Infect Control and Hosp Epidemiol 2004;25:772-7.

2. Kawagoe J.Y., Segre C.A.M., Pereira C.R., et al. Risk factors for nosocomial infections in critically ill newborns: A 5-year prospective cohort study. Am J Infect Control 2001;29:109-14.

3. Zaidi A.K.M., Huskins W.C., Thaver D., et al. Hospital-acquired neonatal infections in developing countries. Lancet 2005;365:1175-88.

4. Brady M.T. Health care-associated infections in the neonatal intensive care unit. Am J Infect Control 2005;33:268-75.

5. Auriti C., Maccalini A., Di Liso G., et al. Risk factors for nosocomial infections in a neonatal intensive-care unit. Journal of Hospital Infection 2003;53:25-30.

6. Lubchenco L.O., Hansman C., Dressler M., Boyd E. Intrauterine growth as estimated from liveborn birth-weight data at 24 to 42 weeks of gestation. Pediatrics 1963;32:793-800.

7. Battaglia F.C., Lubchenco L.O. A practical classification of newborn infants by weight and gestational age. J Pediatr 1967;71:159-63.

8. World Health Organization. Antenatal care randomized trial: Manual for the implementation of the new model. Geneva, 2001; WHO/RHR/01.30-45. 
9. James L.S. Acidosis of the newborn and its relation to birth asphyxia. Acta Paediatr 1960;49 Suppl 122:17-28.

10. Manroe B.L., Weinberg A.G., Rosenfeld C.R., Browne R. The neonatal blood count in health and disease. I. Reference values for neutrophilic cells. J Pediatr 1979;95:89-98.

11. Emori T.G., Culver D.H., Horan T.C., et al. National nosocomial infections surveillance system (NNIS): description of surveillance methodology. Am J Infect Control 1991;19:19-35.

12. Garner J.S., Jarvis W.R., Emori T.G., et al. CDC definitions for nosocomial infections. Am J Infect Control $1988 ; 16: 128-40$.
13. US Department of Health and Human Services. Centers for Disease Control and Prevention. NNIS Manual. Washington, DC: DHHS, 1999.

14. Nagata E., Brito A.S.J., Matsuo T. Nosocomial infections in a neonatal intensive care unit: Incidence and risk factors. Am J Infect Control 2002;30:26-31.

15. Urrea M., Pons M., Serra M., et al. Prospective incidence study of nosocomial infections in a pediatric intensive care unit. Pediatr Infect Dis J 2003;22:490-3.

16. Urrea M., Iriondo M., Thio M., et al. A prospective incidence study of nosocomial infections in a neonatal care unit. Am J Infect Control 2003;31:505-7. 